\title{
Acercar la geodiversidad a través de las salidas de campo en la ESO. Una investigación con el profesorado de ciencias de Bizkaia
}

\section{Approaching geodiversity through field trips in secondary education. A study with science teachers in Biscay.}

T. Zamalloa, G. Maguregi, M. D. Fernández, I. Echevarría

Departamento de Didáctica de la Matemática y de las Ciencias Experimentales, Escuela de Magisterio de Bilbao,

Universidad del Pais Vasco, UPVIEHU

teresa.zamalloa@ehu.es

J. Sanz

Departamento Didáctica de la Matemática y las Ciencias Experimentales. Escuela Universitaria de Magisterio de San Sebastián.

Universidad del Pais Vasco, UPVIEHU

RESUMEN • El presente trabajo explora el perfil académico y la actitud sobre la geología/geodiversidad del profesorado de ciencias de la ESO, analizando las dificultades que retraen a los docentes de realizar salidas de campo y recogiendo las claves positivas de los que sí las realizan. Un estudio llevado a cabo en el territorio histórico de Bizkaia en el que han participado un total de 84 docentes. A través de encuestas y entrevistas, y partiendo de la necesidad de integrar la geodiversidad en el currículo educativo, este proyecto quiere servir, además, para un mayor aprovechamiento de las zonas de alto interés geológico.

PALABRAS CLAVE: geodiversidad; educación secundaria obligatoria; salidas de campo; profesorado; currículo escolar.

ABSTRACT $\bullet$ The present study explores the academic profile and the attitude about geodiversity and geological heritage of the science teachers of obligatory secondary education, analyzing the difficulties of those who do not make field trips and collecting the positive clues of those who do make them. A study performed in Biscay with 84 teachers. Through surveys and interviews and considering the need of the integration of geodiversity in the scholar curriculum, this project wants to be usefull, aswell, to make the most of the high interesting geological areas.

KEYWORDS: geodiversity; secondary school; field trip, faculty; scholar curriculum. 


\section{INTRODUCCIÓN}

\section{La enseńanza de las Ciencias de la Tierra}

Las investigaciones realizadas sobre las actitudes de los estudiantes hacia la ciencia y su desinterés por ésta, tanto en educación primaria (Jarvis y Pell, 2002) como en secundaria (Rose, 2013; Sturman, 2008; TIMSS, 2011), sugieren la modificación de los planteamientos didácticos desarrollados hasta la fecha (Osborne y Dillon, 2008; UNESCO, 2010).

Si bien los niveles de competencia científica, según el Informe PISA (Instituto de Evaluación, 2010), no sitúan a España en una posición alarmante, sí indican un estancamiento en el desarrollo de la competencia científica. Así mismo, el informe ENCIENDE (COSCE, 2011) sugiere la necesidad de fomentar desde el ámbito educativo un cambio didáctico desde metodologías de enseñanza transmisivas y activistas hacia nuevas metodologías más reflexivas y participativas.

Entre los factores que dificultan el aprendizaje de las ciencias se encuentran un lenguaje científico complejo, la inseguridad del profesorado ante enfoques educativos innovadores o una enseñanza que está lejos de los intereses y del ámbito cotidiano del alumnado (Banet, 2007; Cañas y Martín-Díaz, 2010; Izquierdo, 1996). Por tanto, para desarrollar adecuadamente la competencia científica es necesario replantearse dos ámbitos fundamentales, como son «qué se enseña» y "cómo se enseña» (Pedrinaci, 2008).

Los obstáculos para conseguir un aprendizaje significativo de las ciencias en general se acrecientan en el caso de la geología. En este sentido, García (1998) señala la imposibilidad de una observación directa de los fenómenos geológicos, la inmutabilidad de lo que se observa o la incapacidad del alumnado por integrar la geología en el funcionamiento global del planeta. Además, otros autores mencionan la dificultad de comprensión de las escalas de espacio y tiempo (Brusi, Pallí y Mas, 1994; Carrillo, 1998) y la percepción de la geología como una ciencia de contenidos poco atractivos.

En el ámbito de la educación formal se puede hablar también del poco peso de la geología en los contenidos curriculares debido a la subordinación a otras ciencias como la biología o la geografía (Fermeli et al., 2011; Hernández, 2006). A todo ello se suma la escasez de profesores con formación en geología en la impartición del currículo de ciencias, donde se incluyen contenidos de biología, química, geología y física (Calonge, López, Meléndez y Fermeli, 2012).

En cuanto al profesorado, se destaca la inseguridad que siente en la docencia de la geología (King, 2006; Trend, 2000), y manifiesta dificultades en aspectos básicos como los tiempos geológicos (Pedrinaci, 1993). También en la formación universitaria de los futuros maestros se observa el poco tiempo dedicado a la geología en las asignaturas de ciencias y que las prácticas relacionadas con las ciencias de la Tierra son las peor valoradas (Vicente, Vallés y López, 2012).

A todo lo anterior contribuye la paulatina pérdida de peso específico de la geología en el currículo docente no universitario regulado por la LOE, principalmente en la ESO. La geología recibe menos atención que otras disciplinas científicas y se suelen trabajar menos contenidos geológicos en el aula de los que figuran en los programas oficiales (Pedrinaci, 2012b). Como ejemplo de la escasa presencia que puede llegar a tener en los currículos oficiales, en el Boletín Oficial del País Vasco-BOPV (Decreto 175/2007), se determina el currículo de la asignatura de Biología y Geología para el tercer curso de la ESO y en él no aparece ningún contenido de geología. Dado el carácter optativo de las materias de ciencias en cuarto de la ESO, puede haber un porcentaje significativo del alumnado en la Comunidad Autónoma del País Vasco (CAPV) que solo trabaje aspectos geológicos en primero y segundo de la ESO. A estos factores externos se suma la resistencia al cambio en los modelos curriculares en ciencias, "qué, para qué y cómo enseñar, y cómo evaluar» (Sanmartí, Burgoa y Nuño, 2011), que se hace extensivo a la geología. Se concluye, por tanto, que la administración educativa y buena parte del profesora- 
do de ciencias tienen una percepción de que la geología no tiene conexión con la vida cotidiana, y que no interesa ni al alumnado ni al propio profesorado (Pedrinaci, 2012a). Todo ello hace que exista una deriva hacia lo que el anterior autor califica de "círculo vicioso", donde se imparte menos geología que la que está contemplada, lo que conlleva una reducción en los contenidos geológicos en los sucesivos currículos educativos.

Concurren, sin embargo, diversas circunstancias que, adecuadamente gestionadas, pueden tornarse en oportunidades para reorientar y dar importancia a la docencia de las Ciencias de la Tierra.

Por un lado, el cambio que se propone en el modo de enseñar la geología, con una mayor dedicación horaria e incidencia en la necesidad de abordar los conocimientos esenciales de las Ciencias de la Tierra como sistema, incluyendo su relación con la sociedad. Todo ello se condensa en el documento «Alfabetización en Ciencias de la Tierra» (Pedrinaci, 2012a), inspirado en los Earth Science Literacy Principles (NSF, 2009), que se constituyen como una auténtica guía inspiradora en las nuevas formas de entender y enseñar las Ciencias de la Tierra. Se considera, según estos principios, que una persona alfabetizada en Ciencias de la Tierra es aquella que comprende los conceptos y procesos fundamentales de esta disciplina y es capaz de tomar decisiones bien fundadas y responsables sobre la Tierra y sus recursos, entre otros aspectos.

La segunda circunstancia positiva es la creciente socialización de la geología, que incide en la importancia del valor del patrimonio geológico y de la geodiversidad local a través de actuaciones de conservación y catalogación de lugares de interés geológico. Así, se puede encontrar una variedad de espacios que abarcan desde los de mayor valor científico a las iniciativas locales con un enfoque turístico y educativo sin perder la perspectiva lúdica (conjunto paleontológico de Teruel-Dinópolis, yacimientos paleoantropológicos de la Sierra de Atapuerca, Museo del Jurásico de Asturias-MUJA o las georrutas del Alto Tajo, por poner algunos ejemplos). No podemos olvidar los geoparques, espacios singulares tanto por su riqueza científica y ambiental como por sus connotaciones sociales y culturales (como, por ejemplo, el Geoparque de la Costa Vasca, el Parque Cultural del Maestrazgo en Teruel y el Parque Natural del Cabo de Gata-Níjar).

Como indica Carcavilla (2011), todos estos lugares tienen un alto potencial de aprovechamiento como motor de economías locales, pero también un inherente valor educacional, como promueve la Ley de Patrimonio Natural y Biodiversidad (Ley 42/2007).

En este sentido, entre las actuaciones que se están desarrollando en la CAPV se encuentra el inventario de geozonas publicado dentro de la Estrategia de Geodiversidad (Gobierno Vasco, 2011), que ha tenido en consideración para su determinación, entre otros aspectos, su utilización didáctica.

Por tanto, las geozonas pueden constituir un recurso educativo de primer orden, representando de una manera holística los procesos de evolución de la Tierra y de las alteraciones antropogénicas, a través de sus aspectos científicos (biológicos-geológicos), pero también desde las perspectivas de intervención humana en la configuración del paisaje y en la ordenación de los recursos (Belmonte, 2011; Carcavilla, 2007; Martínez-Graña, Goy y Zazo, 2011). Algunos autores identifican que no solo los espacios geológicos especialmente singulares, sino también los hitos geológicos locales, los denominados geotopos, pueden configurarse en herramientas educativas de alto valor (Meléndez, Fermeli, Calonge, Escorihuela y Ramajo, 2011).

Por todo ello, y en el marco de la alfabetización en las Ciencias de la Tierra, uno de los grandes retos es articular adecuadamente estos instrumentos de educación no formal en su integración en la educación formal reglada. Citando a Brusi (1992), en este caso es acertado decir que «los profesores no deben sacar a los alumnos del aula, sino sacar el aula a la calle». 


\section{Las salidas de campo como elemento prioritario de aprendizaje de las Ciencias de la Tierra}

Son muchos los factores que convierten en insustituible el papel didáctico de las salidas de campo, como la mejora de la asimilación de contenidos conceptuales, de procedimientos científicos, así como de actitudes y valores favorables hacia la ciencia, la protección del medio o el trabajo en grupo (García de la Torre, 1991; Pedrinaci, 2012b).

Como indican Jaén y Bernal (1993), la idea de integrar el trabajo práctico en la enseñanza no es nueva, pero no ha sido adecuadamente integrado ni siquiera por los modelos basados en el aprendizaje por descubrimiento o en los de recepción significativa. Como respuesta a los modelos tradicionales (transmisores), los métodos de «aprendizaje por descubrimiento inductivo y autónomo» se aplicaron también a las salidas geológicas (García de la Torre et al., 1993), dando prioridad a la observación desde un enfoque libre de teoría, lo cual tampoco favorece el desarrollo conceptual. El modelo de salida de campo relacionado con la "observación dirigida», entre el modelo transmisivo y el autónomo, puede tender a reproducir errores de ambos si se basa el aprendizaje en el guion de la práctica. Así, tomando como base el constructivismo, numerosos autores abogan por orientar las salidas de campo geológicas mediante el aprendizaje basado en la resolución de problemas (García de la Torre et al., 1993; Pedrinaci, 2012b).

La mayoría de los trabajos desarrollados en las últimas décadas ofrecen claves metodológicas suficientes para planificar y realizar salidas de campo que realmente resulten en un aprendizaje significativo. Sin embargo, a pesar de ello y de la alta valoración que hacen los docentes de las salidas de campo como actividad formativa, es muy significativo que estos mismos admitan que no las realizan de forma habitual (Del Toro y Morcillo, 2011; Pedrinaci, 2012b; Pérez, de Pro y Ato, 2005).

Existen bastantes dificultades tanto pedagógicas como organizativas (Rebelo, Marques y Costa, 2011) para llevar a cabo las salidas de campo, entre estas últimas el excesivo tiempo consumido o el elevado número de alumnado, a las que se unen dificultades del tipo institucional, como la poca flexibilidad de la Administración en relación con este tipo de actividades, la falta de material adecuado y también el poco reconocimiento del valor educativo de los recursos geológicos del entorno.

En cuanto a las dificultades pedagógicas detectadas en varios trabajos (López-Martín, 2007; Pedrinaci, 2012b), se destaca la inseguridad en los propios conocimientos geológicos. Esta inseguridad es a veces una sensación equivocada, y denota una carencia de conocimiento de metodologías adecuadas para el desarrollo de las salidas de campo. Esto coincide con el trabajo realizado por Morcillo, Rodrigo, Centeno y Compiani (1998) sobre un grupo de docentes que admiten, en la mayoría de los casos, diferencias notables entre las salidas que desearían realizar (no dirigidas y de carácter motivadorinvestigativo) frente a lo que realmente hacen, de carácter más bien ilustrativo.

Existen también una serie de carencias actitudinales que pueden tener orígenes diversos. En el caso particular de la geología por el palpable desinterés del alumnado por lo inanimado (Hernández, 2006). Este hecho, junto con la inseguridad antes mencionada y las ideas y experiencias previas del propio profesorado, puede llevar a considerarlas como poco atractivas frente, por ejemplo, a las de biología (Vicente et al., 2012), e incluso a orientar las salidas a trabajar solamente conceptos de biología en sitios de gran valor geológico.

Si bien en los citados trabajos se analizan los aspectos organizativos y funcionales de las salidas de campo geológicas, son escasos los que inciden en los aspectos actitudinales y metodológicos del profesorado (Brusi, Zamorano, Casellas y Bach, 2011), en los que se han detectado grandes carencias y en los que la investigación didáctica puede tener una mayor incidencia.

El presente trabajo ha incidido en el análisis de las dificultades que retraen a los docentes de realizar salidas de campo, así como las claves positivas de los que sí las realizan.

Así mismo, a partir de las opiniones de un grupo de profesorado que no realiza habitualmente salidas de campo geológicas, se han recogido aspectos que hay que tener en cuenta, organizativos y actitudinales, para superar esa resistencia o limitación. 


\section{METODOLOGÍA}

Para este trabajo se ha realizado un cuestionario al profesorado de la ESO de los centros de enseñanza de la provincia de Bizkaia (anexo 1) cuyo esquema general se muestra en la figura 1.

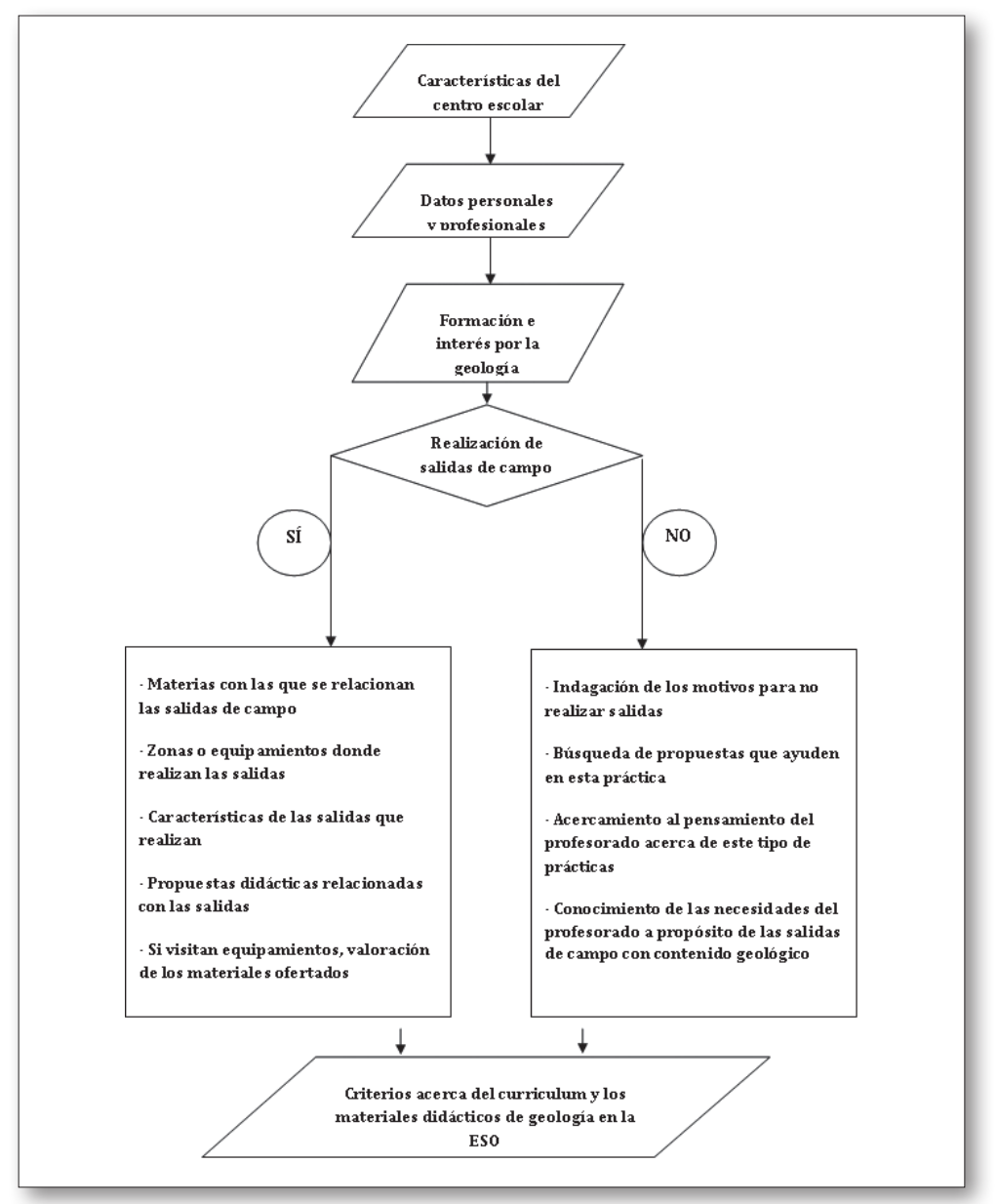

Fig 1. Esquema general de la encuesta.

El cuestionario contiene preguntas cerradas, tipo likert, que permiten tratar las respuestas de modo estadístico, y preguntas más abiertas, con mayor libertad de respuesta, para disponer de información complementaria.

Respecto al sistema de pase de encuestas se utilizaron dos modalidades. Por una parte, la visita a los centros en los que se distribuyeron los cuestionarios entre el profesorado de los seminarios de ciencias. El otro método fue el cuestionario on-line elaborado con la herramienta gratuita y de libre distribución Google Docs.

\section{Consideraciones generales de la muestra}

El total de encuestas recogidas fue de 84 , de las cuales 33 se recogieron telemáticamente y 51 directamente. Estas últimas se recogieron personalmente en 46 de los 173 centros de la provincia de Bizkaia en los que se imparte la etapa de la ESO. 
En un trabajo anterior (Zamalloa, Sanz, Maguregi, Echevarría y Fernández, 2013) se analizó la muestra de la que se extrajo el perfil del profesorado encuestado, que si bien no podemos afirmar que representa a todo el profesorado de la ESO, sí se puede decir que es una aproximación al perfil de este.

De entre las personas encuestadas se observa un número ligeramente mayor de mujeres (48) frente a hombres (33) y, con respecto al rango de edad, está comprendido entre los 30 y los 60 ańos.

Analizando el número de años de experiencia profesional, la mayoría del profesorado se ha dedicado en exclusiva a la docencia durante su vida laboral.

En cuanto a la formación previa del profesorado encuestado, menos del 10\% del profesorado encuestado ha cursado la licenciatura en Geología, más del 60\% la licenciatura en Biología y el 30\% restante, otras licenciaturas. Estos datos, por sí solos, muestran la carencia en formación en Geología del profesorado de ciencias. También desde su propia opinión se confirma esta idea, ya que solamente un $5 \%$ considera que tiene amplios conocimientos sobre la materia, un $40 \%$ afirma que es suficiente y un $52 \%$ declara desear ampliar sus conocimientos.

A pesar de estos datos, un 50\% del profesorado sostiene tener gran interés por la materia a nivel personal, y un $70 \%$ a nivel profesional.

\section{RESULTADOS}

Los resultados se han organizado en cuatro secciones que discurren de acuerdo con los tipos de cuestiones planteadas en el apartado anterior. En cada sección se presentan resultados cuantitativos a cada cuestión, así como resultados cualitativos correspondientes a las preguntas abiertas realizadas al profesorado de la ESO.

\section{¿Realiza salidas de campo el profesorado de Ciencias de la Naturaleza en la ESO?}

La primera cuestión tiene como objetivo saber si el profesorado de Ciencias de la Naturaleza en la ESO realiza o no salidas de campo durante el curso académico. De las 84 respuestas obtenidas, 59 son afirmativas, frente a 25 que son negativas (figura 2). Esto supone que un $70 \%$ del profesorado encuestado utiliza este recurso en su práctica docente.

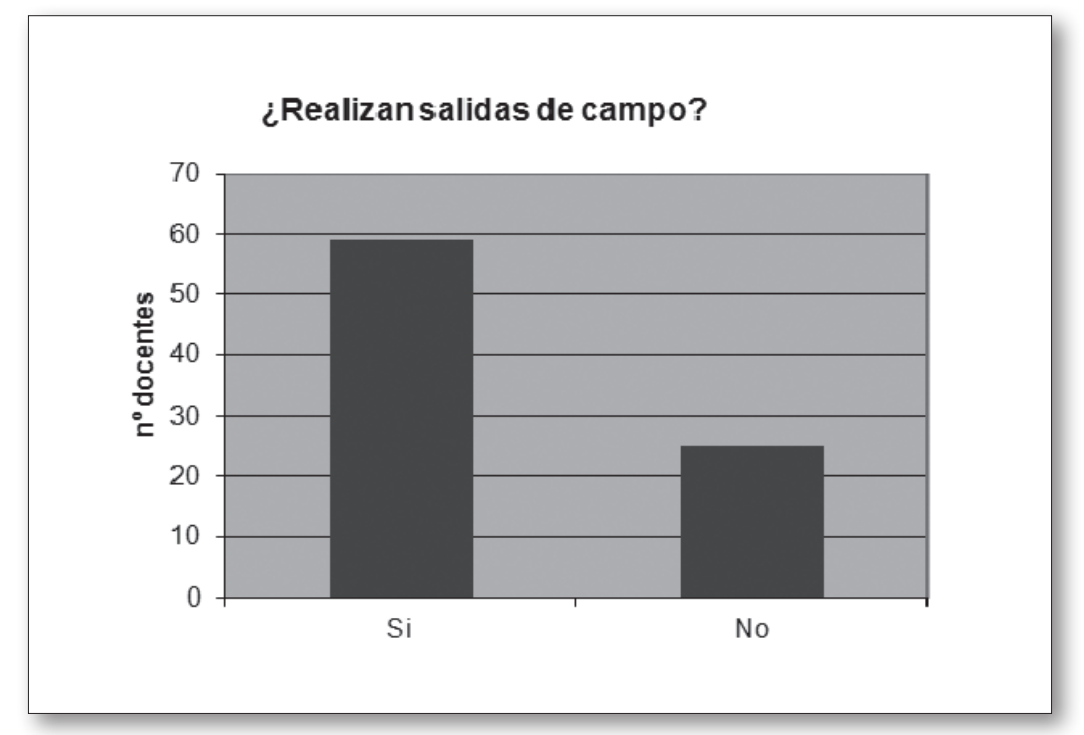

Fig. 2. Profesorado de ciencias de la naturaleza en la ESO que realiza salidas de campo 
A continuación se presentan los resultados de las preguntas relacionadas con el profesorado de ciencias que realiza salidas de campo en su práctica docente.

La figura 3 muestra que en el último curso de la ESO el número de salidas es mayor que en los tres primeros cursos. Esto explica que en la asignatura Biología y Geología se incluyan más salidas de campo que en Ciencias de la Naturaleza (figura 4). Es destacable que dos docentes denominen la asignatura Biología y Geología como Biología, anulando ya en su denominación la parte de geología.

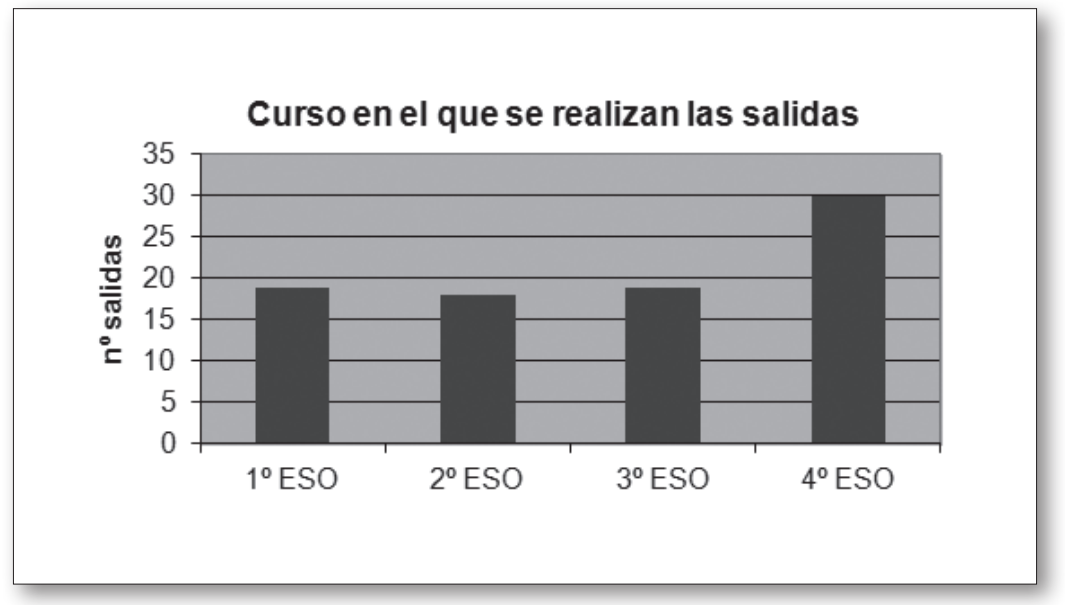

Fig. 3. Cursos en los que se realizan las salidas.

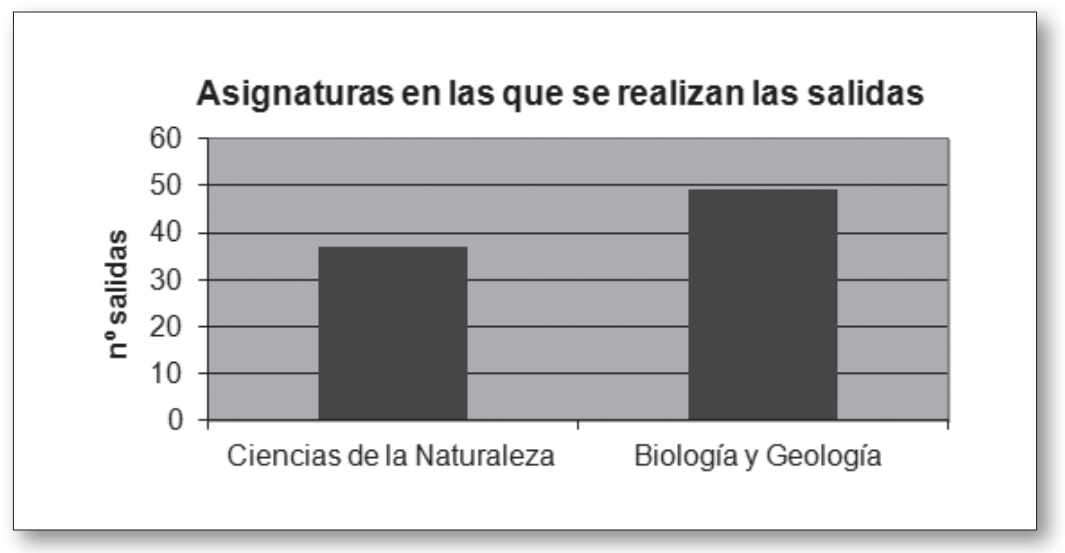

Fig. 4. Asignaturas en las que se realizan las salidas.

El profesorado que incluye salidas de campo en su práctica docente suele hacerlo en varios cursos en los que imparte docencia. Por otro lado, hay que tener en cuenta que la planificación desde el centro de las salidas de campo suele estar distribuida en diferentes materias a lo largo de la ESO.

La pregunta 6 del cuestionario tiene como objetivo conocer los factores que influyen en la elección de salida por parte del profesorado. Los resultados obtenidos (figura 5) ponen de manifiesto que los factores más importantes son una experiencia positiva anterior ( 47 respuestas) y la conexión con el temario trabajado en el aula (43 respuestas). La información publicitada por instituciones educativas de la CAPV tiene un peso inferior pero no despreciable (13 respuestas). 


\section{Factores que influyen en la elección de la salida}

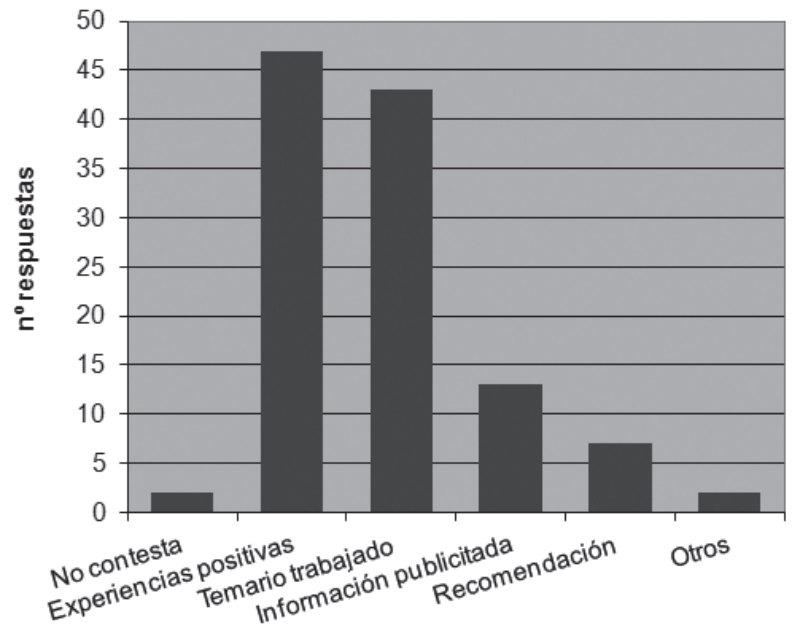

Fig. 5. Factores que influyen en la elección de salida. Nota: La suma de respuestas es superior al $100 \%$, puesto que en algunos casos el propio docente selecciona varios factores.

Al pedir al profesorado que indique los conceptos trabajados durante las salidas, los resultados obtenidos indican que conceptos relacionados con la geología y la biología son mayoritarios frente a conceptos vinculados a física y química (figura 6). Cabe destacar que, aunque el profesorado encuestado es de ciencias, también se trabajan conceptos de ciencias sociales y de historia (relación ciencia-sociedad).

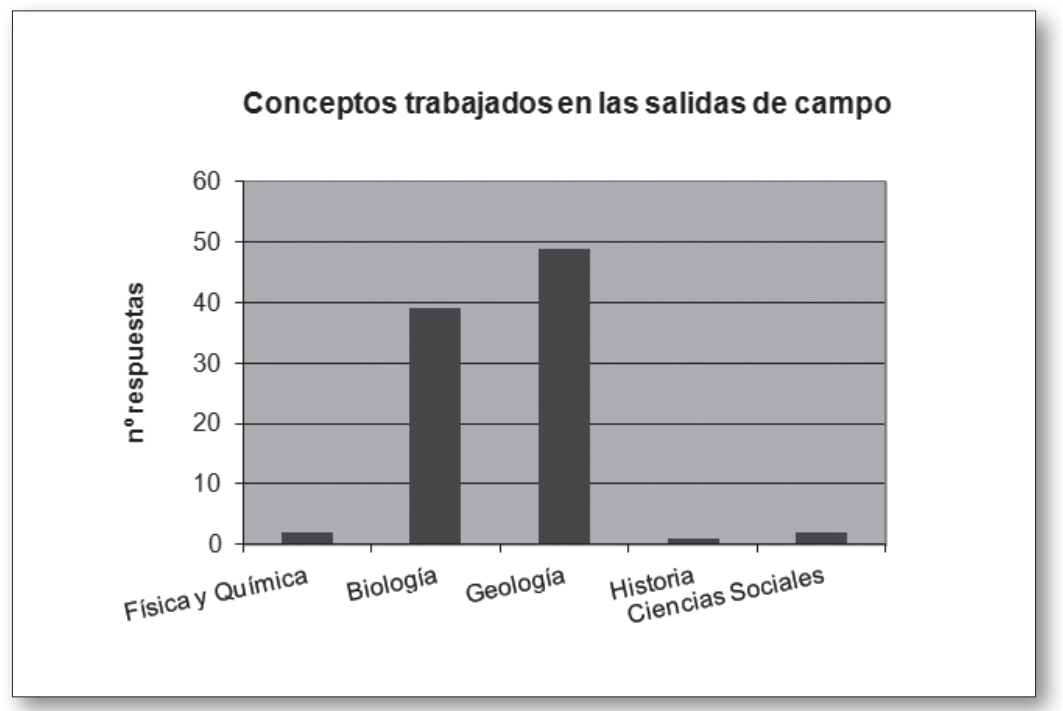

Fig. 6. Conceptos trabajados en las salidas de campo. Nota: La suma de respuestas es superior al $100 \%$, puesto que en algunos casos el propio docente selecciona varios conceptos 
En cuanto a la fuente en la que el profesorado obtiene información acerca de la zona que se va a visitar, 24 docentes de los 58 que realizan salidas no contestan. Esto puede deberse a que la mayoría repite las salidas en años consecutivos (figura 5, experiencias anteriores positivas) y, por tanto, conoce el lugar y no necesita información acerca de la zona. Al analizar las respuestas obtenidas, los folletos informativos e internet son los recursos más utilizados, 40 y 20\% respectivamente (figura 7).

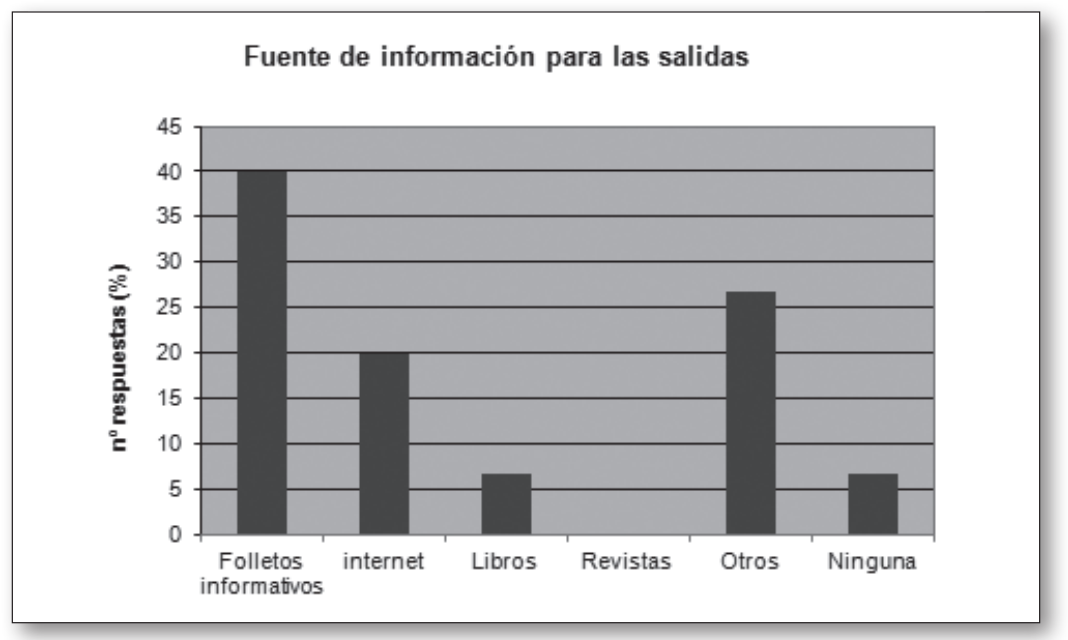

Fig. 7. Fuente de información para la realización de las salidas.

En la figura 8 se muestran los resultados obtenidos respecto a la financiación de estas actividades. La mayor parte de las respuestas (33) indica una financiación mixta. En un 89\% de estos casos la familia es quien, junto al centro u otras instituciones, colabora económicamente en la actividad. Queremos destacar 11 respuestas en las que la financiación es exclusivamente a cargo de las familias y 2 respuestas en las que la familia no está implicada directamente pero sí a través de la Asociación de Madres y Padres del centro (AMPA).

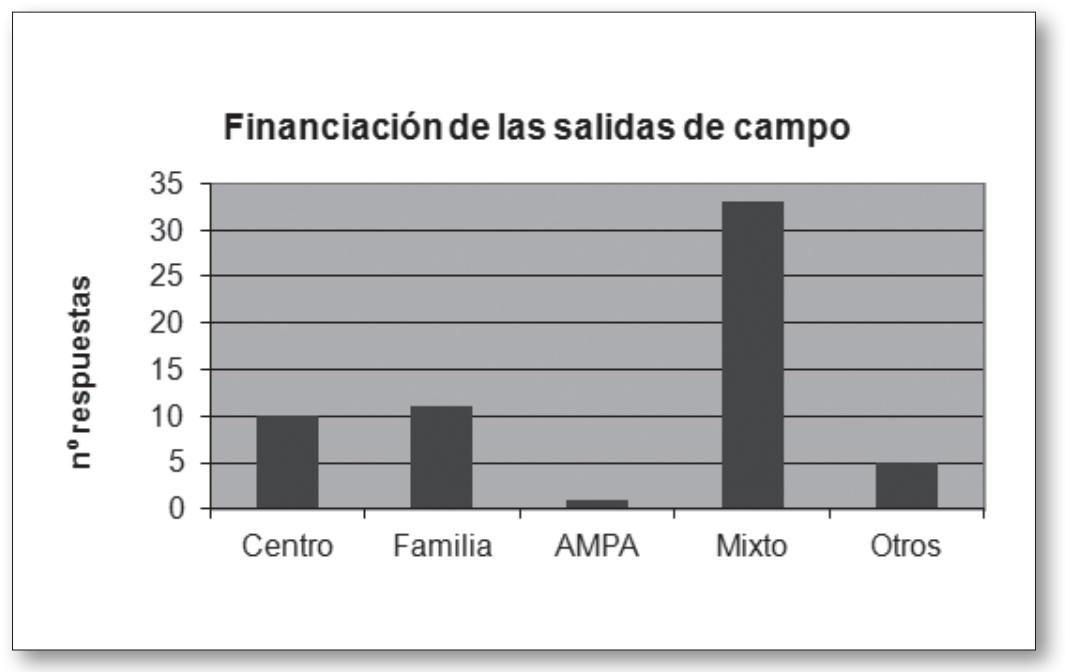

Fig. 8. Financiación de las salidas de campo.

Finalmente, en la pregunta 14 del cuestionario diagnosticamos el grado de satisfacción del profesorado. En una escala del 1 al 5 el valor obtenido fue de 4,07 $\pm 0,62$. Estos datos son coherentes con 
los contenidos en la pregunta 12 del cuestionario, ya que el $97 \%$ del profesorado encuestado tiene intención de repetir la salida con otro alumnado.

\section{¿Cómo se trabajan en el aula las salidas de campo?}

El 97\% del profesorado prepara la visita. En esta preparación destaca la falta de material didáctico tanto para realizar actividades en el aula previas a la visita como para poder llevar a cabo la visita sin necesidad de personal de apoyo que encarece la salida. En la figura 9 podemos apreciar que la actividad más utilizada en la preparación es la explicación previa de contenidos que se van a trabajar en la salida.

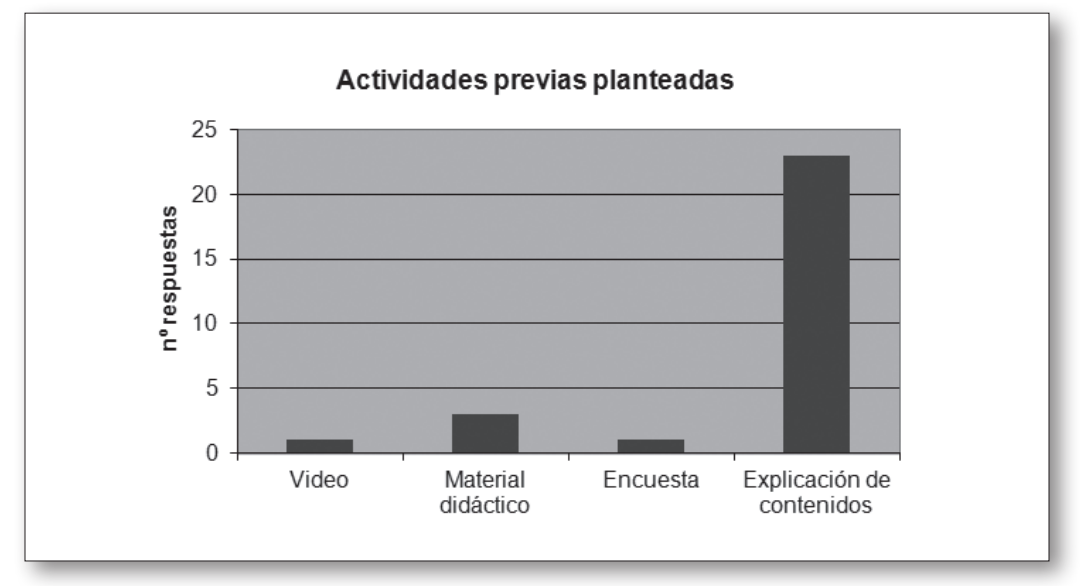

Fig. 9. Actividades previas planteadas a la visita a un equipamiento.

La mayoría del profesorado que realiza visitas (80\%) evalúa al alumnado tras la salida de campo valorando contenidos conceptuales, actitudinales y procedimentales. Para ello se utilizan exposiciones orales (22\%), trabajos individuales escritos (29\%), trabajos en grupo (39\%) y encuestas tipo test $(10 \%)$. Solamente un docente indica que realiza un examen escrito tras la visita en el que solo valora contenidos conceptuales.

\section{Importancia de la geología en las salidas de campo}

El 83\% del profesorado de ciencias de la ESO que realiza salidas de campo afirma que trabaja contenidos geológicos en dichas salidas. En este sentido, los contenidos más trabajados son la geodinámica externa y las rocas y los minerales (figura 10). 


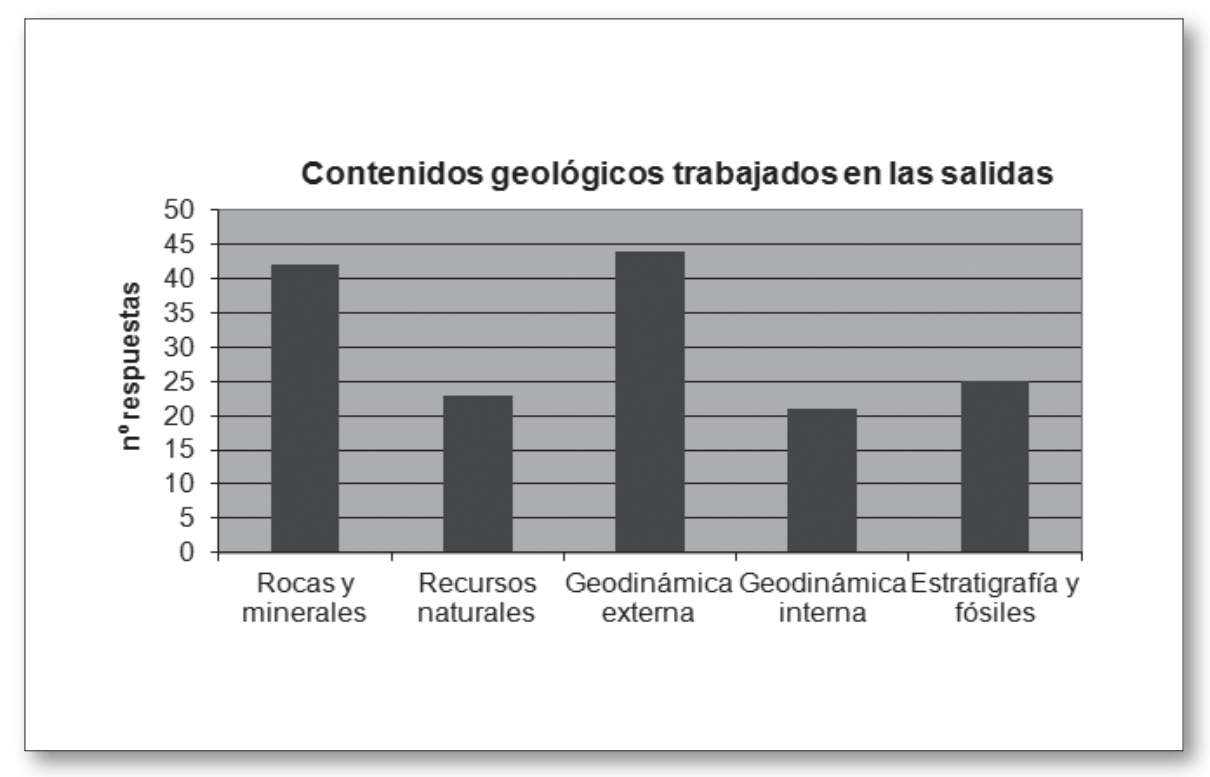

Fig. 10. Contenidos geológicos trabajados en las salidas. Nota: La suma de respuestas es superior al $100 \%$, puesto que en algunos casos el propio docente trabaja varios contenidos geológicos.

Aunque la mayoría del profesorado afirma trabajar contenidos geológicos en las salidas, solamente 13 docentes visitan algún equipamiento que se dedique de manera específica al estudio y comprensión de los georrecursos. El equipamiento más visitado por el profesorado de ciencias de Bizkaia (más del $50 \%$ ) es el centro de interpretación de los recursos naturales Algorri en Zumaia (Gipuzkoa).

En una escala de 1 a 5, el profesorado valora muy positivamente tanto las estrategias de comunicación (carteles y personal) como las explicaciones didácticas y los contenidos geológicos de dichos centros (media \pm EEM: 4,04 \pm 0,92; $4 \pm 0,70 ; 4 \pm 0,95 ; 4,04 \pm 0,75$, respectivamente). Sin embargo, la valoración de las guías escritas es ligeramente inferior (media de 3,42 $\pm 1,15$ ).

\section{¿Cuáles son los motivos para que una parte importante del profesorado de ciencias no rea- lice salidas de campo?}

La mayoría de los docentes (96\%) que no realiza salidas de campo considera que la visita in situ a una zona geológica concreta podría facilitar el aprendizaje significativo del alumnado. Entonces, ¿por qué no realiza salidas de campo?

El principal motivo es la falta de tiempo para integrarlo en el currículo ( $44 \%$ de las respuestas, figura 11), seguido de razones económicas (12\% de las respuestas, figura 11). Otras razones mencionadas (columna otros de la figura 11) aluden a: a) la actitud desfavorable del alumnado en este tipo de actividades; $b$ ) la falta de estabilidad del profesorado interino, lo que implica mayor dificultad para planificar las salidas de campo; c) el escaso peso de la geología en el currículo, y d) el esfuerzo añadido que supone al profesorado organizar una actividad de este tipo. 


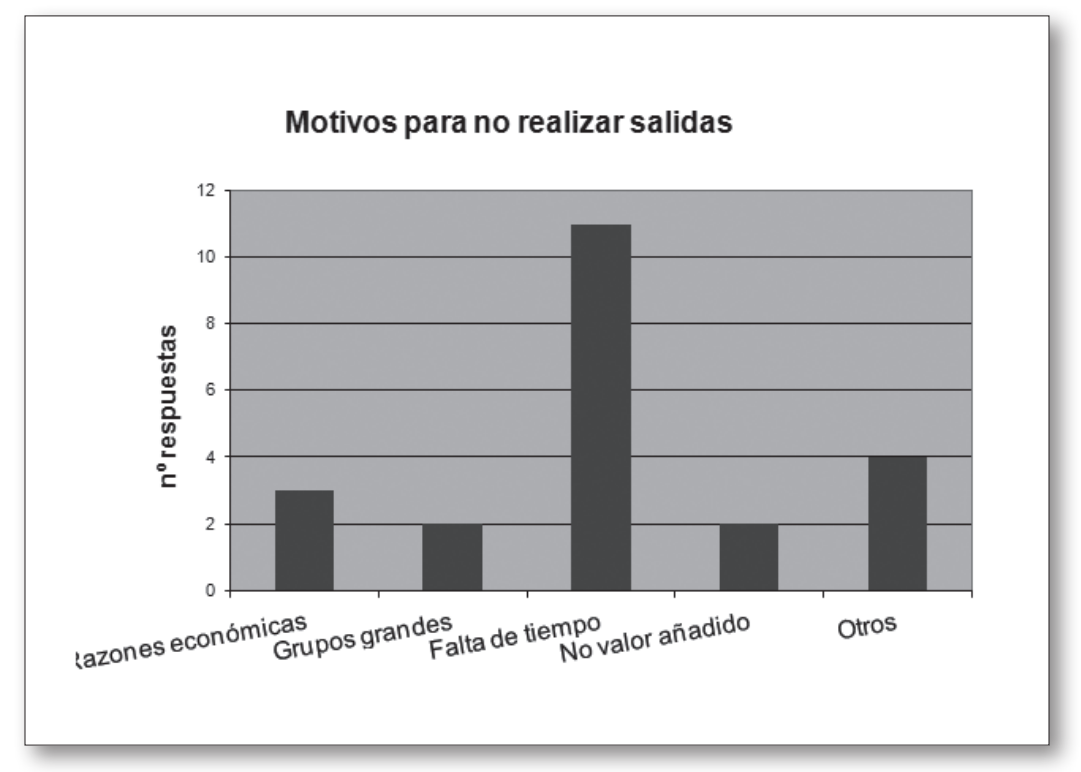

Fig. 11. Motivos para no realizar salidas de campo. El apartado otros incluye respuestas abiertas.

Cabe destacar que un 30\% del profesorado que actualmente no realiza salidas de campo sí que lo ha hecho anteriormente. La razón principal para haber abandonado esta actividad es la movilidad del profesorado interino, tanto entre centros como en la asignación de las materias. Esto da lugar a no conocer con antelación la asignatura que se ha de impartir ni el centro en el que ejercerá su docencia, lo que dificulta la planificación de las salidas de campo con antelación.

El profesorado que no ha realizado nunca salidas de campo (18 docentes) lo consideraría interesante si existiera una oferta atractiva y factible relacionada con la geología/geodiversidad en el entorno de su centro. En estos casos 3 docentes realizarían la visita sin personal de apoyo, mientras que 15 preferirían visitar un centro de interpretación. Es destacable que un porcentaje del profesorado no sabría cómo introducir este tipo de actividades en el currículo (20\% del profesorado encuestado).

La pregunta 20 del cuestionario es una pregunta abierta que tiene como objetivo que el profesorado de la ESO que no realiza salidas de campo indique propuestas de mejora de la oferta de recursos y equipamientos relativos a geología/geodiversidad de la zona para que sean útiles y asequibles como recurso educativo en la ESO. Los resultados cualitativos obtenidos muestran que el profesorado desconoce las actividades existentes en las geozonas de la CAPV, para lo que solicita información, guías escritas, cursos prácticos, material docente y personal de apoyo.

Por último, en la figura 12 se representan los recursos didácticos usados por el profesorado que no utiliza las salidas de campo en su práctica docente. El libro de texto es el recurso utilizado por todos los docentes a excepción de uno ( 24 docentes), seguido de los recursos informáticos (21 docentes). Otro recurso utilizado es el laboratorio de ciencias ( 8 docentes). Sin embargo, las revistas científicas no son un recurso utilizado. 


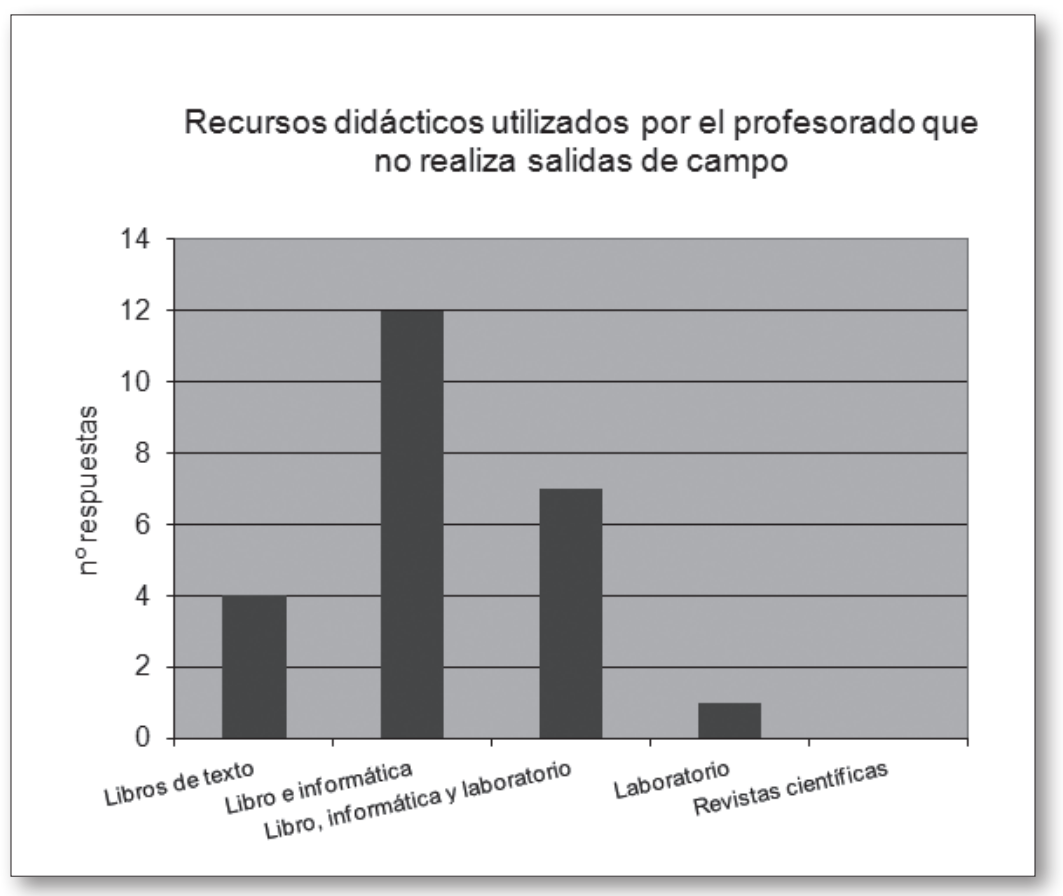

Fig. 12. Recursos utilizados por el profesorado de ciencias que no realiza salidas de campo.

\section{CONCLUSIONES}

Las conclusiones obtenidas se presentan estructuradas teniendo en cuenta las preguntas de investigación realizadas.

\section{¿Realiza salidas de campo el profesorado de ciencias en la ESO?}

La mayoría del profesorado encuestado realiza salidas de campo como recurso educativo en la ESO. Se realiza un mayor número de salidas en cuarto curso, que es cuando se imparte la asignatura Biología y Geología, frente a los cursos en los que se imparte la asignatura Ciencias de la Naturaleza. Esto puede ser debido a que esta última comprende materias como física y química además de biología y geología. Además, la dedicación horaria a la asignatura es como máximo de 3 horas por semana, lo que dificulta la realización de salidas por la dedicación de tiempo que estas suponen (Decreto 175/2007). Sin embargo, cabe destacar que se realizan salidas de campo en los cuatro cursos de la ESO. El hecho de que, a pesar del escaso tiempo semanal asignado, el profesorado de la ESO realice salidas de campo muestra la consideración de estas como un recurso educativo importante en la enseñanza-aprendizaje de las ciencias (Brusi, 1992; García de la Torre, Sequeiros y Pedrinaci, 1993).

El factor que más influye en la realización de las salidas es haber tenido una experiencia positiva anterior, lo cual puede estar relacionado con la satisfacción del alumnado desde los puntos de vista tanto cognitivo como actitudinal (Falk, 1983; Meredith, Fortner y Mullins, 1997).

La segunda de las causas es que el contenido de la salida esté integrado en el temario, siendo esta una de las claves para que formen parte del aprendizaje significativo (Del Toro y Morcillo, 2011) y se alcancen los objetivos educativos. 
Además, durante las salidas de campo se trabajan sobre todo contenidos de geología, así como de biología. Sin embargo, también en estas salidas se incluyen contenidos relacionados con la enseñanzaaprendizaje de las Ciencias Sociales. Esto supone que se abordan desde una perspectiva contextualizada (Jaén y Bernal, 1993), teniendo en cuenta su relación con los fenómenos sociales, tal como propone Pedrinaci $(2012 b)$.

Además, las salidas son financiadas mayoritariamente por las familias, de manera directa o a través del AMPA. A pesar de las dificultades que esta situación entraña, el grupo de profesores y profesoras investigados realiza salidas de campo en todos los cursos de la ESO. Esto debería subsanarse estableciendo una mayor partida presupuestaria por parte de la Administración educativa para financiar este tipo de actividades.

\section{¿Cómo se trabajan en el aula las salidas de campo?}

La preparación de la visita, junto con la evaluación posterior, es, según algunos autores, uno de los aspectos que tiene mayor influencia en el éxito de la salida, pero en el que en ocasiones menos se incide (Brusi et al., 2011). Si bien todo el profesorado que realiza salidas de campo las prepara, realizando actividades previas, estas consisten, fundamentalmente, en la explicación de los contenidos que se van a trabajar durante la salida de una manera transmisiva y sin evaluar en la mayoría de los casos los conocimientos del alumnado.

Como indican Garcia de la Torre et al. (1993) es adecuado conocer previamente lo que el alumnado realmente sabe y determinar qué conceptos clave necesita conocer y qué principios geológicos fundamentales necesita saber, en especial en lo relativo a las edades geológicas. Pero, además, Orion y Hofstein (1994) constatan que existe una relación directa entre la preparación de la visita por parte del alumnado y el aprendizaje que ha realizado e incluso con el nivel de satisfacción de esta. Como resumen Morcillo et al. (1998) definen el novelty factor ('factor de novedad'), que se basa en el nivel de aprendizaje previo, entrenamiento con el área de salida y aspectos psicológicos (empoderamiento de la metodología). En esa misma línea, el análisis realizado por Brusi et al. (2011) incide en que han de trabajarse previamente los objetivos de la visita, los aspectos logísticos e incluso la recopilación de información. Se mejoran así los aspectos metodológicos y actitudinales, pasando de una actividad ilustrativa a una salida de campo motivadora.

El profesorado encuestado alude en muchos casos a una falta de material educativo para trabajar en el aula antes de la realización de la salida de campo e incluso para poder realizar la salida sin la necesidad de monitores, con el consiguiente encarecimiento de la actividad. En muchos casos se ha destacado la ausencia de materiales específicos, por lo que la fuente de información principal son los propios libros de texto de la enseńanza reglada, o la información informal facilitada por otros colegas (King, 2006). Se debe, por tanto, fomentar la búsqueda de materiales específicos de la zona (Catana y Caetano, 2009; Simón, Catana y Poch, 2011), haciendo partícipe al alumnado, o desarrollando materiales propios que contemplen aspectos conceptuales, procedimentales y actitudinales (Moreira, Praia y Sofré, 2002). Otros aspectos como los cursos formativos (Aramburu, 1993) o una mayor incidencia en los grados o másteres de profesorado (Del Toro y Morcillo, 2011) completarían la preparación del profesorado (Morcillo et al., 1998).

Así mismo, la mayoría del profesorado evalúa al alumnado una vez realizada la salida, teniendo en cuenta los diferentes tipos de contenidos. Esto se corresponde con las propuestas metodológicas que realizan diversos autores (Cantó, Hurtado y Vilches, 2013; Pedrinaci, 2012b; Vilaseca y Bach, 1993), que plantean actividades no solo durante la salida y previamente a ella, sino también una vez realizada. El profesorado encuestado en este trabajo menciona que las herramientas de evaluación utilizadas fueron los trabajos, en grupo e individuales, que pueden ser más efectivas que otras herramientas más 
tradicionales (exámenes...) para evaluar actividades de esta índole (Rebelo et al., 2011). Se evalúan, así, no solo competencias relacionadas con la interpretación de problemas geológicos o la comunicación de las conclusiones, sino también competencias transversales como el trabajo en grupo, el respeto ambiental y la conservación del patrimonio geológico (Brusi et al., 2011).

Se puede concluir que para superar las dificultades mencionadas sería necesario elaborar materiales didácticos sobre los lugares que reúnan un mayor interés educativo y que suelen ser visitados con mayor frecuencia, para ser utilizados en la fase anterior, durante la salida y posteriormente, con el alumnado de ESO, mejorando a su vez algunos aspectos metodológicos y conceptuales.

\section{¿Qué importancia tiene la geología en las salidas de campo?}

Otro de los factores que influye en la realización de salidas de campo es la conexión con el temario que se trabaja durante el curso. Además, la gran mayoría del profesorado que realiza salidas de campo trabaja contenidos de geología. En particular, contenidos de geodinámica externa y rocas y minerales, que son algunos de los que se plantean en el currículo de la ESO (Decreto 175/2007).

El equipamiento más visitado por el profesorado investigado, del territorio de Bizkaia, es el Centro de Interpretación de Algorri, que ofrece visitas guiadas al Flysch de Zumaia (Gipuzkoa). Por un lado, la denominación de Geoparque en 2010 supone contar con el reconocimiento y la difusión de la información de un enclave que reúne un importante patrimonio geológico. Por otro lado, este centro de interpretación es de los pocos, si no el único, en la CAPV que proporciona una oferta educativa centrada en contenidos geológicos. Se puede afirmar, por tanto, que el profesorado manifiesta interés en conocer esta geozona, aunque esté alejada de su entorno más cercano, porque pone a su disposición una interesante propuesta didáctica que puede relacionar con los contenidos trabajados en el aula.

Es por ello por lo que se debería diversificar la oferta educativa centrada en contenidos de Ciencias de la Tierra a otros enclaves de interés geológico (Carcavilla, 2011).

\section{¿Cuáles son los motivos para que una parte importante del profesorado de ciencias no realice salidas de campo?}

El profesorado que no realiza salidas de campo también las considera un recurso importante para la enseñanza-aprendizaje de las ciencias. Este resultado coincide con el realizado por Cortés et al. (2012), donde maestros en formación, después de cursar las asignaturas correspondientes, identifican como aspectos fundamentales en su formación aspectos didácticos y metodológicos (entre ellos las salidas de campo), sobre los aspectos teóricos, identificados inicialmente.

Sin embargo, son numerosas las razones manifestadas para no incluirlas como actividades en el desarrollo del programa de sus asignaturas, así como variadas las propuestas de mejora que plantean. Por lo que para facilitar que el profesorado salga al campo sería necesario tenerlas en cuenta. Entre ellas estarían la de proporcionar formación sobre la geodiversidad de la CAPV, su valor educativo y su inserción en los programas curriculares de la ESO. Así mismo, se deberían diseñar materiales didácticos para su utilización en el aula y ofertar cursos prácticos de formación.

El reto que se presenta es complejo, pero si se consideran las salidas de campo como un recurso imprescindible en la enseñanza-aprendizaje de las ciencias, como formadores de profesorado debemos abordarlo. 


\section{AGRADECIMIENTOS}

Proyecto subvencionado por la Universidad del País Vasco UPV/EHU (NUPV11/20)

\section{REFERENCIAS BIBLIOGRÁFICAS}

Aramburu, C. (1993). Resultados de una encuesta sobre cursos de actualización en geología para el profesorado de enseñanzas primaria y secundaria. Enseñanza de las Ciencias de la Tierra, 1 (2), pp. 113-120.

Banet, E. (2007). Finalidades de la educación científica en secundaria: opinión del profesorado sobre la situación actual. Enseñanza de las Ciencias, 25 (1), pp. 5-20.

Belmonte, A. (2011). Apadrina un P.I.G.: El patrimonio geológico como recurso didáctico. Enseñan$z a$ de las Ciencias de la Tierra, 19 (2), pp. 210-214.

BOE (2007). Ley 42/2007, del 13 de diciembre, del Patrimonio Natural y de la Biodiversidad, 299, pp. $51275-51327$.

Brusi, D. (1992). Reflexiones en torno a la Didáctica de las salidas de campo en Geología I: Aspectos funcionales. II: Aspectos metodológicos. Actas VII Simposio sobre Enseñanza de la Geología. Santiago de Compostela, pp. 363-407.

Brusi, D.; Pallí, L. y Mas, J. (1994). El espacio y el tiempo en Geología. Enseñanza de las Ciencias de la Tierra. Número extra. Actas del VIII Simposio de Enseñanza de la Geología. Córdoba, pp. 44-45.

Brusi, D.; Zamorano, M.; Casellas, R.M. y Bach J. (2011). Reflexiones sobre el diseño por competencias en el trabajo de campo en Geología. Enseñanza de las Ciencias de la Tierra, 19 (1), pp. 4-14.

Calonge, A., López, M.D., Meléndez, G. y Fermeli G. (2012) Geoschools, el reto de mejorar la enseñanza de la Geología en la educación secundaria europea. En A. M. Sarmiento, M. Cantano y G. R. Almodovar (Eds.), Actas del XVII Simposio sobre Enseñanza de la Geología, Huelva: Universidad de Huelva publicaciones, pp. 48-53

Cantó, J.; Hurtado, A. y Vilches, A. (2013). Educación científica más allá del aula. Una herramienta para la formación del profesorado en sostenibilidad. Alambique, 74, pp. 76-82.

Cañas, A. y Martín-Díaz, M.J. (2010). ¿Puede la competencia científica acercar la ciencia a los intereses del alumnado? Alambique, 66, pp. 80-87.

Carcavilla, L. (2007). La divulgación de la Geología en espacios protegidos: Las geo-rutas del Parque Natural del Alto Tajo (Guadalajara). Enseñanza de las Ciencias de la Tierra, 15 (1), pp. 65-76.

Carcavilla, L. (2011). Geoturismo y geoconservación: amenazas y oportunidades. Avances y retos en la conservación del patrimonio geológico en España. En E. Fernández-Martínez y R. Castaño de Luis (eds.). Avances y retos en la conservación del Patrimonio Geológico en España. Actas de la IX Reunión Nacional de la Comisión de Patrimonio Geológico (Sociedad Geológica de España). León: Área de Publicaciones, Universidad de León, pp. 31-38.

Carrillo, L. (1998). Ciencias de la Tierra y Matemática ¿tendrá descendencia esta pareja? Aula de Innovación Educativa, 69. Disponible en línea: http://www.grao.com/revistas/aula/069-los-materialesen-ciencias-experimentales--como-ve-las-matematicas-el-profesorado-de-otras-areas/ciencias-dela-tierra-y-matematicas-tendra-descendencia-esta-pareja. (Última consulta, 26 de noviembre de 2013).

Catana, M.M. y Caetano, M.I. (2009). Los programas educativos para escuelas del Geopark Naturtejo (Portugal): Un aprendizaje en el campo. Enseñanza de las Ciencias de la Tierra, 17 (1), pp. 93-101. 
COSCE-Confederación de Sociedades Científicas de España (2011). Informe ENCIENDE. Enseñanza de las Ciencias en la Didáctica Escolar para edades tempranas en España. Madrid: COSCE. Disponible en línea: http://www.cosce.org/pdf/Informe_ENCIENDE.pdf. (Última consulta, 18 de julio de 2013).

Cortés, A.L.; Gándara, M.; Calvo, J.M.; Martínez, M.B.; Gil, M.J., Ibarra, J. et al. (2012). Expectativas, necesidades y oportunidades de los maestros en formación ante la enseńanza de las Ciencias en la Educación Primaria. Enseñanza de las Ciencias, 30 (3), pp. 155-176.

Decreto 175/2007, de 16 de octubre, por el que se establece el currículo de la Educación Básica y se implanta en la Comunidad Autónoma del País Vasco. (BOPV 218, de 13 de noviembre de 2007).

Del Toro, R. y Morcillo, J.G. (2011). Las actividades de campo en educación secundaria. Un estudio comparativo entre Dinamarca y España. Enseñanza de las Ciencias de la Tierra, 19 (1), pp. $39-47$.

FALK, J.H. (1983). Field trips: A look at environmental effects on learning. Journal of Biological Education, 17 (2), pp. 137-142.

Fermeli, G.; Meléndez, G.; Calonge, A.; Dermitzakis, M.; Steininger, F.; Koutsouveli, A. et al. (2011). Geoschools: innovative teaching of Geosciences in secondary schools and raising awareness on geoheritage in the society. En E. Fernández-Martínez y R. Castaño de Luis (eds.). Avances y retos en la conservación del Patrimonio Geológico en España. Actas de la IX Reunión Nacional de la Comisión de Patrimonio Geológico (Sociedad Geológica de España). León: Área de Publicaciones, Universidad de León, pp. 120-124.

García, C.M. (1998). De los obstáculos epistemológicos a los conceptos estructurantes: una aproximación a la enseñanza-aprendizaje de la geología. Enseñanza de las Ciencias, 16 (2), pp. 323-330.

García de la Torre, E. (1991). Recursos en la enseñanza de la Geología: la Geología del campo. Investigación en la escuela, 13, pp. 85-96.

García De La Torre, E.; Sequeiros, L. y Pedrinaci, E. (1993). Fundamentos para el aprendizaje de la Geología de campo en Educación Secundaria: Una propuesta para la formación del profesorado. Enseñanza de las Ciencias de la Tierra, 1 (1), pp. 11-18.

Gobierno VAsco (2011). Geoturismo sostenible en la red de espacios naturales protegidos de la Comunidad Autónoma del País Vasco, fase 2.2. Evaluación de geozonas, valoración del grado de cumplimiento de los requisitos del manual de la red GEO-Basque. Vitoria-Gasteiz: Servicio de Publicaciones del Gobierno Vasco.

Hernández, M.J. (2006). Panorámica actual sobre las prácticas de geología en el ámbito escolar. Alambique, 47, pp. 30-37.

Instituto de Evaluación (2010). PISA 2009. Programa para la Evaluación Internacional de los Alumnos. OCDE Informe español. Madrid: Ministerio de Educación. Disponible en línea: http://iaqse. caib.es/documents/aval2009-10/pisa2009-informe-espanol.pdf (Última consulta, 26 de noviembre de 2013)

IzQuiErdo, M. (1996). Reflexiones sobre el lenguaje científico: algunos ejemplos de Geología. Enseñanza de las Ciencias de la Tierra, 4 (3), pp. 167-171.

JaÉn, M. y Bernal, M. (1993). Integración del trabajo de campo en el desarrollo de la enseñanza de la Geología. Enseñanza de las Ciencias de la Tierra, 1 (3), pp. 153-158. 
Jarvis, T. y Pell, A. (2002). Changes in primary boys' and girls' attitudes to school and science during a two-year in-service programme. The Curriculum Journal, 13(1), pp. 43-69.

http://dx.doi.org/10.1080/09585170110115268

King, C. (2006). Enseñar geología a los profesores de Ciencias: la experiencia de la Earth Science Education Unit (ESEU). Enseñanza de las Ciencias de la Tierra, 14 (2), pp. 142-149.

Ley 42/2007, de 13 de diciembre, del Patrimonio Natural y de la Biodiversidad. BOE, 299, de 14 de diciembre de 2007.

López-Martín, J.A. (2007). Las salidas al campo: mucho más que una excursión. Revista Educar en el 2000: Revista de Educación ambiental, 11, pp. 100-103.

Martinez-Graña, A.M.; Goy, J.L. y Zazo, C. (2011). Natural Heritage Mapping of the Las Batuecas-Sierra de Francia and Quilamas Nature Parks (SW Salamanca, Spain). Journal of Maps, 7 (1), pp. 600-613.

Meléndez, G.; Fermeli, G.; Calonge, A.; Escorinuela, J. y Ramajo, J. (2011). Using geological heritage as a useful educational tool in secondary schools: the Geo-schools Project and the use of educational geotopes. En E. Fernández-Martínez y R. Castaño de Luis (eds.). Avances y retos en la conservación del Patrimonio Geológico en España. Actas de la IX Reunión Nacional de la Comisión de Patrimonio Geológico (Sociedad Geológica de España). León: Área de Publicaciones, Universidad de León, pp. 191-195.

Meredith, J.E.; Fortner, R.W. y Mullins, G.W. (1997). Model of affective learning for nonformal science education facilities. Journal of Research in Science Teaching, 34(8), pp. 805-817. http://dx.doi.org/10.1002/(SICI) 1098-2736(199710)34:8<805::AID-TEA4>3.0.CO;2-Z

Morcillo, J.G.; Rodrigo, M.; Centeno, J.D. y Compiani M. (1998). Caracterización de las prácticas de campo: justificación y primeros resultados de una encuesta al profesorado. Enseñanza de las Ciencias de la Tierra, 6 (3), pp. 242-250.

Moreira, J.; Praia, J. y Sofré, F. (2002). La construcción de materiales didácticos en geología de campo: un estudio sobre alumnos de enseñanza secundaria. Enseñanza de las Ciencias de la Tierra, 10 (2), pp. 185-192.

NSF-National Science Fundation (2009). New Report Sets Forth Principles of Earth Science Literacy. Disponible en línea: http://www.nsf.gov/news/news_summ.jsp?cntn_id=114904. (Última consulta: 26 de noviembre de 2013).

Orion, N. y Hofstein, A. (1994). Factors that influence learning during a scientific field trip in a natural environment. Journal of Research in Science Teaching, 31 (10), pp. 1097-1119. http://dx.doi.org/10.1002/tea.3660311005

Osborne, J. y Dillon, J. (2008). Science Education in Europe: Critical Reflections. Londres: Nuffield Foundation.

Pedrinaci, E. (1993). La construcción histórica del tiempo geológico. Enseñanza de las Ciencias, 11 (3), pp. 315-323.

Pedrinaci, E. (2008). ¿Tiene sentido una materia como las ciencias para el mundo contemporáneo? Enseñanza de las Ciencias de la Tierra, 16 (1), pp. 9-16.

Pedrinaci, E. (2012a). Alfabetización en Ciencias de la Tierra, una propuesta necesaria. Enseñanza de las Ciencias de la Tierra, 20 (2), pp. 133-140.

Pedrinaci, E. (2012b). Trabajo de campo y aprendizaje de las ciencias. Alambique, 71, pp. 81-89.

Pérez, A.; De Pro, A. y Ato, M. (2005). Evaluación nacional de actitudes y valores hacia la ciencia en entornos educativos. Madrid: FECYT.

Rebelo, D., Marqués, L. y Costa, N. (2011). Actividades en ambientes exteriores al aula en la Educación en Ciencias: contribuciones para su operatividad. Enseñanza de las Ciencias de la Tierra, 19(1), pp. 15-25. 
Rose (2013). The Relevance of Science Education. Disponible en línea: www.roseproject.no. (Última consulta, 26 de noviembre de 2013).

Sanmartí, N.; Burgoa, B. y Nuño, T. (2011). ¿Por qué el alumnado tiene dificultad para utilizar sus conocimientos científicos escolares en situaciones cotidianas? Alambique, 67, pp. 62-69.

Simón, J.L.; Catana, M.M. y Poch, J. (2011). La enseñanza de la Geología en el campo: un compromiso de los Geoparques reconocidos por la UNESCO. Enseñanza de las Ciencias de la Tierra, 19 (1), pp. 74-80.

Sturman, L. (2008). Maths, science and gender: messages from TIMSS. Practical Research for Education, 40, pp. 68-75.

TIMSS (2011). TIMSS 2011 International Results in Science. Disponible en línea: http://timssandpirls.bc.edu/timss2011/international-results-science.html. (Última consulta, 26 de noviembre de 2013).

TREnd, R. (2000). Conceptions of geological time among primary teacher trainees, with reference to their engagement with geoscience, history, and science. International Journal of Science Education, 22 (5), pp. 539-555. http://dx.doi.org/10.1080/095006900289778

UNESCO (2010). Current Challenges in Basic Science Education. París: UNESCO Education Sector.

Vicente, F.; Vallés, C. y López, M. ${ }^{a} A$. (2012). Aportaciones a la formación científica desde actividades prácticas en el Grado de Maestro de Educación Primaria. En J.M. Domínguez Castañeira (ed.). Actas de XXV Encuentro de Didáctica de las Ciencias Experimentales. Santiago de Compostela: Universidad de Santiago de Compostela, pp. 1313-1320.

Vilaseca, A. y Bach, J. (1993). ¿Podemos evaluar el trabajo de campo? Enseñanza de las Ciencias de la Tierra, 1 (3), pp. 158-167.

Zamalloa, T.; Sanz, J.; Maguregi, G.; Echevarría, I. y Fernández, M.D. (2013, septiembre). Perfil académico y actitud sobre la Geología/Geodiversidad en el profesorado de ciencias de la ESO. Comunicación presentada al IX Congreso Internacional sobre Investigación en Didáctica de las Ciencias. Girona, Espańa. Disponible en línea: http://congres.manners.es/congres_ciencia/gestio/ creacioCD/cd/articulos/art_951.pdf. (Última consulta: 26 de noviembre de 2013). 


\section{ANEXO I.}

\section{Encuesta}

\section{i. Centro escolar}

Financiación:

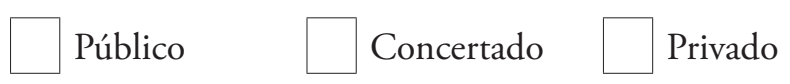

N. ${ }^{\circ}$ alumnos

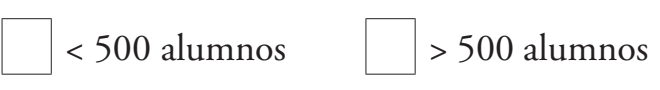

\section{Datos personales}

Fecha de nacimiento:

Sexo:

Licenciatura en:

Años de experiencia docente:

Asignaturas que impartes en la actualidad y curso:

\section{FORMACIÓN PREVIA}

1. ¿Tienes formación específica relacionada con la geología? Sí/No. Indica qué tipo.

2. En una autoevaluación de tus conocimientos en geología, ¿cómo te definirías?

\begin{tabular}{|l|l|}
\hline Con amplios conocimientos & \\
\hline Con conocimientos suficientes & \\
\hline Me gustaría tener un mayor conocimiento & \\
\hline Otros & \\
\hline
\end{tabular}

3. En una autoevaluación de tu interés en geología/geodiversidad, ¿cómo te definirías?

\begin{tabular}{|l|l|}
\hline Muy interesado desde el punto de vista profesional & \\
\hline Interesado desde el punto de vista personal & \\
\hline Interesado desde el punto de vista profesional & \\
\hline Preferiría incidir en otros conocimientos para mi docencia & \\
\hline Otros & \\
\hline
\end{tabular}

\section{Salidas de campo}

4. ¿Realizas salidas de campo a lo largo del curso?

$$
\text { Sí }
$$

$\mathrm{NO}$

En caso negativo, continúa contestando la encuesta a partir de la pregunta 15 .

En caso afirmativo contesta las preguntas 5 a 14: 
5. Indica por favor en qué curso y asignatura realizas las salidas, así como los conceptos trabajados

\begin{tabular}{|c|c|c|c|}
\hline Curso & Asignatura & Concepto/s trabajado/s & Lugar/es \\
\hline & & & \\
\hline & & & \\
\hline
\end{tabular}

6. ¿Por qué motivos has elegido esa zona/ese equipamiento para la realización de la salida?

\begin{tabular}{|l|l|}
\hline Por experiencias positivas anteriores & \\
\hline Por estar relacionada con el temario que estabas trabajando en el aula & \\
\hline Por información publicitada por instituciones educativas de la CAPV & \\
\hline Por recomendación de algún/a compañero/a & \\
\hline Otros & \\
\hline
\end{tabular}

7. ¿En qué fuentes has obtenido información acerca de la zona?

\begin{tabular}{|l|l|}
\hline Folletos informativos & \\
\hline Internet & \\
\hline Libros & \\
\hline Revistas & \\
\hline Otras & \\
\hline Ninguna & \\
\hline
\end{tabular}

8. ¿Qué has echado en falta tanto para la preparación como para la gestión de la visita?

9. ¿Planteas alguna actividad previa a la visita al equipamiento?

¿Cuál?

SÍ

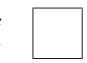

NO

\begin{tabular}{|l|l|}
\hline Visualización de un vídeo & \\
\hline Actividades propuestas en los materiales del equipamiento & \\
\hline Encuesta escrita para detectar conocimientos previos & \\
\hline Explicación de contenidos que se trabajarán en la salida & \\
\hline Otros & \\
\hline
\end{tabular}

10. ¿Evalúas al alumnado una vez realizada la salida?

a) ¿Cómo lo haces?

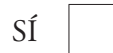

$\mathrm{NO}$

\begin{tabular}{|l|l|}
\hline Exposición oral & \\
\hline Trabajo individual escrito & \\
\hline Trabajo en grupo & \\
\hline Encuesta tipo test & \\
\hline Otros & \\
\hline
\end{tabular}


b) ¿Qué aspectos tienes en cuenta para la evaluación?

\begin{tabular}{|l|l|}
\hline Contenidos conceptuales & \\
\hline Contenidos actitudinales & \\
\hline Contenidos procedimentales & \\
\hline
\end{tabular}

11. La financiación de estas actividades es con cargo:

\begin{tabular}{|l|l|}
\hline Al centro & \\
\hline A las familias & \\
\hline A la asociación de padres & \\
\hline Mixto (familia, centro...) & \\
\hline Otros & \\
\hline
\end{tabular}

12. ¿Repetirás la salida con otro alumnado?

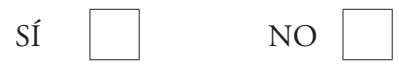

13. ¿Trabajas contenidos geológicos en las salidas?

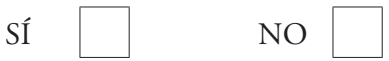

En caso afirmativo:

a) ¿Qué contenidos geológicos trabajas en las salidas?

\begin{tabular}{|l|l|}
\hline Rocas y minerales & \\
\hline Recursos naturales & \\
\hline Geodinámica externa & \\
\hline Geodinámica interna & \\
\hline Estratigrafía y fósiles & \\
\hline Otros & \\
\hline
\end{tabular}

b) ¿Visitas algún equipamiento que se dedique de manera específica al estudio y comprensión de los georrecursos?, ¿`cuál?

c) En caso de que sea afirmativa tu respuesta a la pregunta anterior, expresa tu valoración de los siguientes apartados (1 es la puntuación mínima y 5 la máxima):

\begin{tabular}{|c|}
\hline Estrategias de comunicacio \\
\hline Carteles y paneles explicativos \\
\hline Guías escritas y panfletos \\
\hline Personal en caso de visita guiada \\
\hline Explicaciones didácticas \\
\hline Adecuadas al nivel de comprensión del visitante \\
\hline Contenidos geológicos \\
\hline Reflejan la riqueza geológica de la zona \\
\hline
\end{tabular}


14. Respecto a la satisfacción en general con la/s salida/s, expresa tu valoración del 1 al 5 (1 es la puntuación mínima y 5 la máxima):

\begin{tabular}{|l|l|}
\hline Satisfacción personal del profesor & \\
\hline Satisfacción del alumnado & \\
\hline
\end{tabular}

Por favor, continúa rellenando la encuesta a partir de la pregunta 21 (apartado 4)

(Viene de la pregunta 4):

15. ¿Cuál es el motivo principal para no realizar salidas de campo? Marca con una X la casilla correspondiente

\begin{tabular}{|l|l|}
\hline Razones económicas & \\
\hline Grupos demasiado grandes de alumnos & \\
\hline Falta de tiempo para integrarlo en el currículo & \\
\hline No conozco recursos cercanos al centro escolar & \\
\hline No creo que aporten ningún valor añadido a las clases & \\
\hline Otros & \\
\hline
\end{tabular}

16. Aunque actualmente no realices salidas de campo, ¿’has realizado alguna anteriormente?

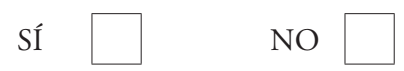

a) En caso afirmativo,

¿Cuál?

¿Por qué no la has vuelto a realizar?

Marca con una X la casilla correspondiente

\begin{tabular}{|l|l|}
\hline No resultó tan interesante como esperaba & \\
\hline Las instalaciones/equipamientos no eran adecuados & \\
\hline Razones económicas & \\
\hline Grupos demasiado grandes de alumnos & \\
\hline Falta de tiempo para integrarlo en el currículo & \\
\hline Ya no imparto la materia correspondiente & \\
\hline Otros & \\
\hline
\end{tabular}

b) En caso negativo, si existiera una oferta atractiva y factible relacionada con la geología/ geodiversidad en el entorno de tu centro consideras que una salida de campo sería:

Marca con una X la casilla correspondiente

\begin{tabular}{|l|l|}
\hline Interesante & \\
\hline No sabría cómo introducirlo en el currículo & \\
\hline No creo que aportara ningún valor añadido a las clases & \\
\hline No tendríamos medios para realizarla & \\
\hline Otros & \\
\hline
\end{tabular}


¿Cómo organizarías/elegirías la visita?

Marca con una X la casilla correspondiente

\begin{tabular}{|l|l|}
\hline Serías tú el guía & \\
\hline Preferirías visitar un georrecurso que contase con un centro de interpretación & \\
\hline Te gustaría tener información previa & \\
\hline Preferirías apoyarte en los contenidos del centro y trabajar después en clase & \\
\hline Visitarías un georrecurso de tu entorno próximo $(<30 \mathrm{~km})$ & \\
\hline Visitarías un georrecurso situado a una distancia $>30 \mathrm{~km}$ & \\
\hline
\end{tabular}

17. ¿Consideras que la visita in situ a una zona geológica concreta podría facilitar el aprendizaje significativo del alumnado?

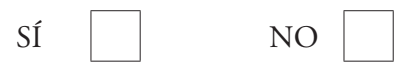

En caso de responder negativamente indica por qué...........

18. Marca con una $\mathrm{X}$ los diferentes recursos didácticos que utilizas en tus clases de ciencias a la hora de explicar geología

\begin{tabular}{|l|l|}
\hline Libros de texto & \\
\hline Revistas científicas & \\
\hline Laboratorio de ciencias & \\
\hline Recursos informáticos & \\
\hline Prácticas de aula & \\
\hline Seminarios & \\
\hline Otros & \\
\hline
\end{tabular}

19. ¿Conoces algún georrecurso susceptible de visita en la CAPV? ¿Cuál?

20. ¿Qué propondrías para mejorar la oferta de recursos y equipamientos relativos a geología/ geodiversidad de la zona, para que fueran útiles y asequibles para el profesorado de la ESO? 


\title{
Approaching geodiversity through field trips in secondary education. A study with science teachers in Biscay
}

\author{
T. Zamalloa, G. Maguregi, M. D. Fernández, I. Echevarría \\ Departamento de Didáctica de la Matemática y de las Ciencias Experimentales, \\ Escuela de Magisterio de Bilbao, \\ Universidad del País Vasco, UPV/EHU \\ teresa.zamalloa@ehu.es \\ J. Sanz \\ Departamento Didáctica de la Matemática y las Ciencias Experimentales. \\ Escuela Universitaria de Magisterio de San Sebastián. \\ Universidad del País Vasco, UPV/EHU
}

Different analysis performed in the last years show a loss of weight of Geology in the compulsory education curriculum and a decrease of students and teachers' interest. This can be due to numerous factors such as the perception of Geology as an immobile science with unattractive contents, or a lack of teachers with specific training in Geology, whose insecurity can lead to a reduction of hours dedicated to this topic. These attitudes may have motivated the administration to reduce Geology contents in previous curricula.

This situation is significantly confronted with the increasing socialization of Geology and the rise of promotion and diffusion of geodiversity experienced over the last years, which is, in itself, a potential instrument of informal education. Almost anywhere in Spain, cataloguing and interpretation initiatives of sites with special geologic interest can be found, from a scientific, touristic, patrimonial or ludic point of view. Not only the bestknown and most singular sites but also the local landmarks hold an inherent educational value.

Therefore, one of the challenges to make Geology teaching more attractive may be the integration of local geodiversity in the formal education. One of the aspects that shall be promoted are field trips to these local sites. In this regard, just visiting the visitor's centre does not assure a significant educational experience, as some other aspects should be necessarily considered, such as: a) the cooperative work before and after the field trip, b) the evaluation of procedural, attitudinal and conceptual contents, c) the incorporation of social or environmental aspects or d) the integration of the field trip in the subject of the course, among others.

Taking the lack of teachers' preparation in terms of Geology as a hypothesis, the present work has evaluated the integration of geodiversity in the scholar curriculum and the attitude towards field visits. The research was carried out through surveys and interviews to secondary education science teachers from Biscay (Spain).

Among some of the results, only $10 \%$ of the teachers have a specific training in Geology, although the majority indicates that they would like to increase their knowledge. Therefore, an interest in the topic is noticed.

A $70 \%$ of the survey respondents have participated in field trips related with Geology, most of them in the $4^{\text {th }}$ course of compulsory secondary education. The rest of them have not carried out any field trips mainly due to a lack of time or to economic reasons.

It should be pointed out that the teachers who consider field trips plan them properly, including previous preparation of the students and different post-field trip assessment activities. However, many teachers agree on the lack of educational material for the preparation of the trip or even make the visit on their own with no guides or instructors.

Finally, it is also significant that the most visited facility is the Basque Coast Geo-park interpretation center, located in the nearest province of Gipuzkoa. This could account for the attractive and complete didactic proposal offered by the center, which complements the contents worked in the classroom, as well as the social recognition of the area.

These results highlight the potential of local geodiversity and the sites related to it as a suitable tool for teaching Geology. Any geological offer should include appropriate educational contents, along with teachers' training to make visits efficient and meaningful learning experiences. 
